\title{
Value Comparison of Iterative Reconstruction Algorithm-Based Chest CT and Ultrasound in the Diagnosis of Community-Acquired Pneumonia in Children
}

\author{
Lijuan Wu $\mathbb{D}^{1},{ }^{1}$ Jianwei Ji $\mathbb{D}$, ${ }^{1}$ and Yunlong Shi $\mathbb{C}^{2}$ \\ ${ }^{1}$ Department of Pediatrics, Yiwu Central Hospital, Yiwu 322000, China \\ ${ }^{2}$ Department of Neonatology, Yiwu Central Hospital, Yiwu 322000, China \\ Correspondence should be addressed to Jianwei Ji; 20200120876@nxmu.edu.cn
}

Received 16 December 2021; Revised 17 January 2022; Accepted 20 January 2022; Published 21 February 2022

Academic Editor: M Pallikonda Rajasekaran

Copyright (c) 2022 Lijuan Wu et al. This is an open access article distributed under the Creative Commons Attribution License, which permits unrestricted use, distribution, and reproduction in any medium, provided the original work is properly cited.

\begin{abstract}
This study aimed to explore the diagnostic value of chest computed tomography (CT) based on adaptive statistical iterative reconstruction ( $\mathrm{ASiR}$ ) in the optimal weight range for children with community-acquired pneumonia (CAP) and find the differences between chest CT and ultrasound in the diagnosis of CAP. 106 children who were diagnosed as CAP were selected as the research objects. All objects underwent CT and ultrasound scans to measure the noise of CT images after iterative reconstruction and the contrast-to-noise ratio (CNR) of the thoracic aorta and the back muscles. The correlation between ASiR weight, noise, and CNR was analyzed and compared further. In addition, the differences in imaging manifestations of lung consolidation, abnormal pleural line, alveolar interstitial syndrome, and pleural effusion were observed under the ultrasound and CT scans of the patients. The sensitivity, specificity, positive predicted value (PV), and negative PV of the CT scan and ultrasound were analyzed further for the diagnosis of CAP in children. The results showed that ASiR weight and image noise were extremely and negatively correlated $(P<0.001)$ and that CNR and ASiR weight were extremely and positively correlated $(P<0.001) .40-60 \%$ of ASiR image noise was in the middle position, and the muscle space and subcutaneous fat in CT images were clearer when ASiR was at $40 \%$ and $60 \% .60 .38 \%$ of children were diagnosed as CAP. The proportions of children with lung consolidation, interstitial changes, pleural effusion, and atelectasis after ultrasound and CT examination were not greatly different $(P>0.05)$. After ASiR, the sensitivity of CT in detecting the abnormal pleural line + B-line/consolidation feature was the highest, $93.75 \%$. The results of ultrasound diagnosis were consistent with those of chest CT diagnosis $(P<0.01)$. This indicated that the sensitivity, specificity, positive PV, and negative PV of ASiR-based chest CT in the diagnosis of CAP were close to those of ultrasound.
\end{abstract}

\section{Introduction}

Community-acquired pneumonia (CAP) in children is an infectious parenchymal inflammation of the lung accompanied by pathogen infection, which is infected outside of a health care facility and in the incubation period, and is developed during the average incubation period after hospitalization [1]. CAP is the main cause of death for children under 5 years of age and seriously threatens the life and health of children [2]. At present, the CAP is mainly diagnosed clinically with the chest CT, chest X-ray, and lung ultrasound. Among them, pulmonary ultrasound is widely used in clinical diagnosis due to its nonradiation, low cost, and easy operation. However, the value of applying it to the diagnosis of CAP is still being confirmed, and the ultrasound results lack intuitive imaging performance [3]. Chest $\mathrm{CT}$ and chest $\mathrm{X}$-ray examinations cause radiation and have a certain impact on the health of children.

With the development of medicine and the continuous updating of multislice spiral CT technology, the application range of $\mathrm{CT}$ in clinical diagnosis is becoming wider and wider. In order to increase the quality of CT images, the amount of radioactive agents is also increasing, which further increases the risk of cancer caused by radiation. The CT scanning process is accompanied by low-dose ionizing radiation, which eventually leads to point mutations in the 
body's deoxyribonucleic acid (DNA) and chromosomal ectopic, which stimulate the occurrence of tumors [4]. In order to minimize ionizing radiation, some scholars have proposed the concept of low-dose CT (LDCT) scanning. At present, the commonly used low-dose measures include adjusting the width of the $\mathrm{X}$-ray beam, using noise reduction filters, and minimizing the exposure range [5]. However, the current CT scan based on the filtered back projection (FBP) algorithm will cause the increase of CT image noise after adopting low-dose measures [6]. Studies have pointed out that [7] the ASiR not only can ensure the quality of CT images, but also has the potential to reduce the CT radiation dose. At present, the ASiR has been widely used in chest, abdomen, and CT angiography, but whether this algorithm can obtain the exact CT value is inconclusive.

To sum up, this study adopted the ASiR algorithm for the diagnosis of children with CAP and selected 106 children, who were diagnosed as CAP in the Respiratory Department of Yiwu Central Hospital from September 2018 to December 2019 , as the research objects to analyze the impacts of radiation dose and ASiR on lung CT images of children with CAP. In addition, the results were compared with the diagnosis results of ultrasound to explore the diagnostic value of chest CT in CAP of children based on the optimal weight range of $\mathrm{ASiR}$ and its difference from ultrasound imaging, so as to provide reliable reference to apply the ASiR algorithm to the lung CT reconstructed image clinically.

\section{Materials and Methods}

2.1. Research Objects and Grouping. In this study, 106 children who were diagnosed as CAP in the hospital from September 2018 to December 2020 were selected as the research objects, including 59 boys and 47 girls with the age range of 6 months -3 years old. The inclusion criteria were defined as follows: the children had completed ultrasound and chest CT examinations within 48 hours; the children underwent ultrasound and CT examinations blindly. The exclusion criteria were determined as follows: children with severe immunosuppression; children who failed to complete the ultrasound and CT examinations within 48 hours; children with chronic lung disease; children with basic lung or cardiovascular disease; newborns. This study had been approved by the hospital ethics committee, and guardians of all subjects included in the study had signed the informed consent forms.

\subsection{CT Scanning and Image Reconstruction Algorithm.} Each child was scanned in a supine position with a computerized tomography device in a quiet state for enhanced chest scan. The scanning layer thickness and layer distance were both $5 \mathrm{~mm}$, the pitch was set to $0.984: 1$, the collimation thickness was $0.625 \mathrm{~mm} \times 64$, and the tube voltage was set to $100 \mathrm{kVp}$. After the CT scan was completed, the collected data was reconstructed with the ASiR algorithm (with a weight range of 10-100\%), and the obtained mediastinal window and thin-slice lung window images were transmitted to GE Healthcare AW4.4 software for analysis. The reconstructed image noise and CNR were measured and calculated by the same experienced radiologist. The CNR could be calculated with the following equation:

$$
\mathrm{CNR}=\frac{\mathrm{ROI}_{a}-\mathrm{ROI}_{b}}{S D} .
$$

Here, SD refers to the average value of fat density in the left axillary area of interest, $\mathrm{ROI}$ represents the CT value of the thoracic aorta, and $\mathrm{RO}_{b}$ represents the CT value of the muscle. All the above data had to be repeated 3 times, and the average value was calculated and utilized. In addition, the Compare software was adopted to ensure that the size and location of the area of interest were at the same level.

2.3. Evaluation Method and Scoring Criteria of the CT Image Quality. Quality of all images was evaluated by two radiologists with many years of experience in the AW4.4 software alone, and the Compare software was utilized to ensure that all the areas of interest observed by CT images were at the same level. The double-blind method was employed to evaluate the image quality of the mediastinal window and the lung window. In addition, the CT image was scored subjectively according to the European guidelines [8]. The scoring criteria were as follows: (1) Lobes interval, network structure, bronchial vascular bundles, and nodules were visible under the lung window. (2) The pericardial and mediastinal blood vessels, the contrast of lymph nodes and back muscles, and the clarity of muscle tissue can be observed under the mediastinal window. (3) Contrast, organization level, and fineness of the image were visible. A 6point system was used for scoring [9]: 1 point if the image was blurred and cannot be diagnosed; 2 points if the image quality was not good with serious impact on the result of the diagnosis; 3 points if the quality structure of the image was severely blurred and it was difficult to give accurate judgments; 4 points if the quality structure of CT image was moderately fuzzy and the diagnosis judgment was limited; 5 points if the image quality structure was slightly fuzzy without effect on the diagnosis result; 6 points if the image structure was clear without any effect on the diagnosis result.

2.4. Ultrasound Inspection and Judgment Method. During the ultrasound examination, the children were placed in the prone or supine position in a quiet state. The ultrasound probe started from the second intercostal space, scanned along the intercostal space, and then was rotated by $90^{\circ}$ for longitudinal scanning. The total inspection time was controlled within 8-15 minutes. At the same time, the pleural effusion, abnormal pleural line, and pulmonary consolidation of the children were observed and recorded, and the imaging performance was determined according to the ultrasound imaging standard [10]. Pleural effusion referred to the presence of fluid with a width greater than $3 \mathrm{~mm}$ in the pleural cavity; pulmonary consolidation referred to uninflated lungs with or without inflatable bronchial signs; and abnormal pleural line referred to the blurring, interruption, thickening, or even disappearing of the pleural line. 
2.5. Imaging Evaluation Standard. The diagnostic performances of CT and ultrasound in children with CAP were mainly evaluated in terms of sensitivity, specificity, positive $\mathrm{PV}$, and negative PV [11]. The calculation equations could be expressed as follows: $S=(T P / T P+F N)$, $S_{f}=(T N / T N+F P), \quad P P V=(T P / T P+F P), \quad$ and $N P V=(T N / F N+T N)$. Among them, $S, S_{f}, P P V$, and $N P V$ represent the sensitivity, specificity, positive $\mathrm{PV}$, and negative $\mathrm{PV}$, respectively; $T P$ represents true positive; $F N$ refers to false negative; $T N$ represents true negative; and FP represents false positive.

2.6. Statistical Methods. The test data was processed using SPSS19.0 statistical software. The measurement data was expressed as mean \pm standard deviation $(\bar{x} \pm s)$, and count data was indicated as percentage (\%). The counted data was analyzed with $\chi^{2}$ test. The CNR, SD, and scores of lung window and mediastinal window were given in the form of mean \pm standard deviation $(\bar{x} \pm s)$, and their differences were analyzed by single-factor variance. The correlation between ASiR weight and noise and CNR was analyzed by Pearson's product moment analysis. For all data results, $P<0.05$ indicated that the difference was statistically significant.

\section{Results}

3.1. Correlation Analysis of CT Image Noise, CNR, and ASiR Weight. After the ASiR algorithm was employed to reconstruct the CT images of the children, the correlation between the ASiR weight of the CT image and the image noise was analyzed, and the result is shown in Figure 1. It reveals that as the ASiR weight of the CT image increased, the image noise showed a continuous downward trend, which means that the ASiR weight had an extremely negative correlation with the image noise $(r=0.8671$, and $P<0.001)$. In addition, when the ASiR weight was $10 \%$, the image noise reached the maximum value $(29.87 \pm 2.93 \mathrm{HU})$; moreover, the image noise reached the minimum value $(10.07 \pm 1.54 \mathrm{HU})$ when the ASiR weight was $100 \%$.

After the CT image of a child was reconstructed with the ASiR algorithm, the correlation between the ASiR weight and the image CNR was analyzed further, and the result is shown in Figure 2. It illustrates that the CNR value of CT images increased with the increase in the weight of ASiR. Therefore, the CNR value was observably and positively correlated with the ASiR weight $(r=0.917$, and $P<0.001)$. In addition, the CNR value of the image was the smallest $(6.95 \pm 1.92 \mathrm{HU})$ when the ASiR weight was $10 \%$, and the CNR value of the image reached the maximum $(17.23 \pm 4.41 \mathrm{HU})$ when the ASiR weight was $100 \%$.

\subsection{The Correlation of Subjective Scores of Lung Window and} Mediastinal Window with ASiR Weight. After reconstructing a child's CT image using the ASiR algorithm, the correlation of the subjective scores of lung window and mediastinal window with the ASiR weight was analyzed, as given in Figure 3. Figure 3 shows that the image quality of

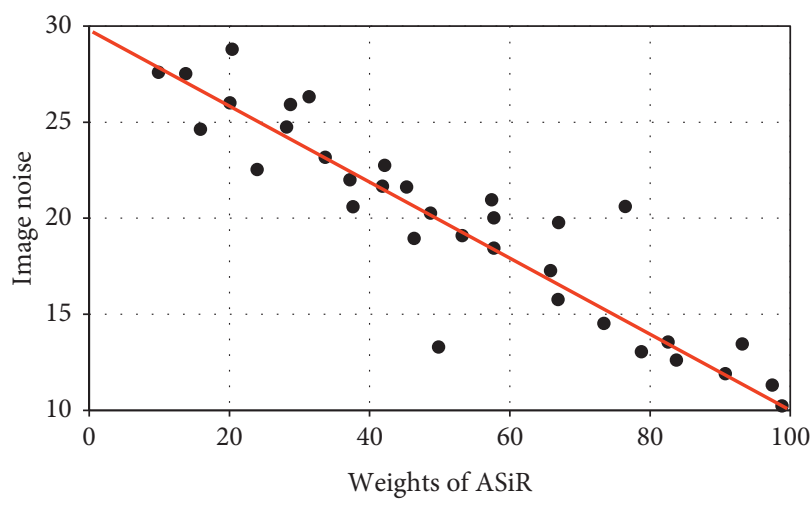

FIgURE 1: The correlation between the ASiR weight of the CT image and the image noise.

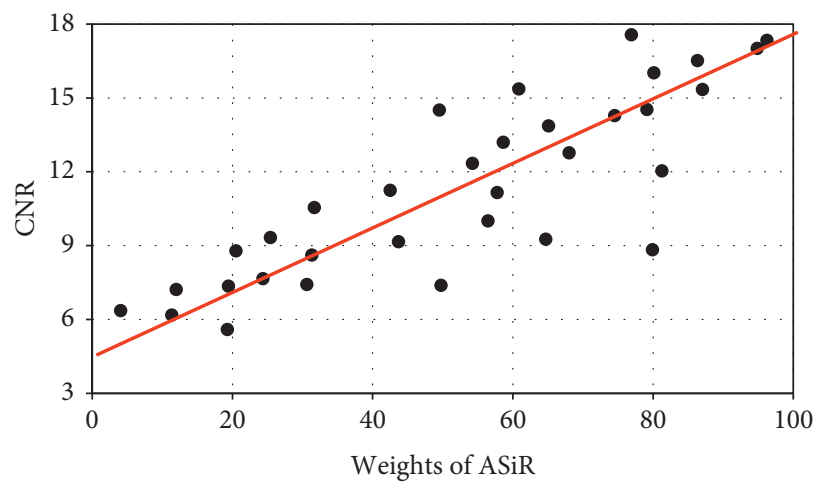

FIgURE 2: The correlation between the ASiR weight and the image CNR.

the lung window and the mediastinal window indicated a trend of increasing and then decreasing with the continuous increase of the ASiR weight. When the ASiR weight was less than $50 \%$, the image quality of the lung window and the mediastinal window showed a continuously increasing trend as the ASiR weight increased. When the ASiR weight was higher than $50 \%$, the image quality reflected a downward trend. When the ASiR weight was higher than $50 \%$, the subjective score of the image was also lower as the ASiR weight increased. When the ASiR weight was higher than $60 \%$, the subjective scores of lung window and mediastinal window were both lower than 5 points; the subjective score $s$ of lung window and mediastinal window were lower than 3 when the ASiR weight was greater than $90 \%$, which could not meet the diagnostic requirements. When the ASiR weight was $40-60 \%$, the scores of the lung window and the mediastinal window were both higher than 5 . At this time, the image noise was relatively low, and the image quality was high.

3.3. CT Images under Various ASiR Weights. CT images under $20 \%, 40 \%, 60 \%, 80 \%$, and $100 \%$ of ASiR weight were observed and analyzed. The results shown in Figure 4 reveal that the streak artifacts and graininess of CT image were getting smaller and smaller as the weight of ASiR continued to increase. When the ASiR was $40 \%$ and $60 \%$, the muscle 


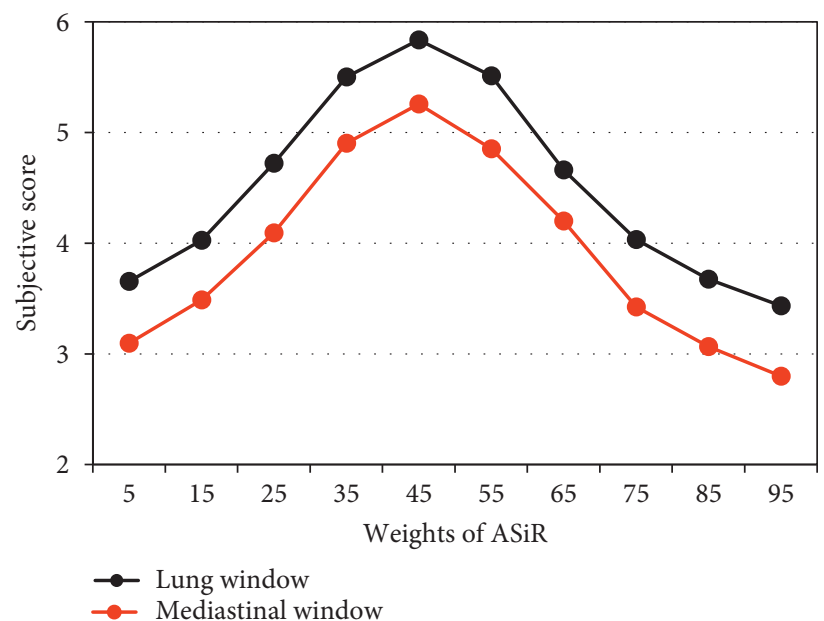

FIgUre 3: The correlation of the subjective scores of lung window and mediastinal window with the ASiR weight.

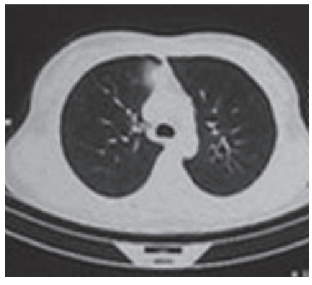

(a)

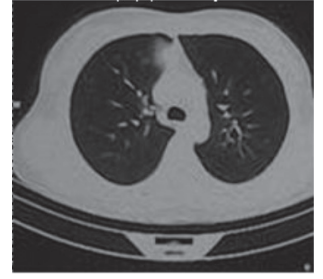

(b)

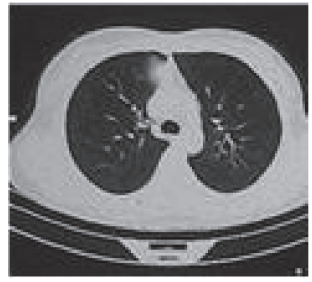

(c)

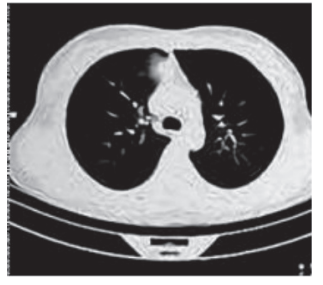

(d)

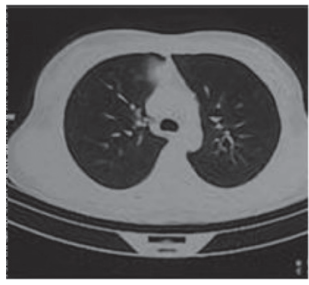

(e)

FIgUre 4: CT images under different ASiR weights: (a) 20\%, (b) 40\%, (c) 60\%, (d) $80 \%$, and (e) $100 \%$.

space and subcutaneous fat of the CT image could be displayed clearly. However, when ASiR was $80 \%$ and $100 \%$, the intermuscular space and subcutaneous fat were too blurred.

3.4. General Information of Children. The general information of the children showed that the average age of the children was $1.02 \pm 0.98$ years old. The age distribution of the children shown in Figure 5(a) illustrated that the children aged 29 days to 3 months accounted for the highest percentage $(33.02 \%)$, followed by the children aged 3-6 months $(24.53 \%)$ and the children aged $1-2$ years $(5.66 \%$, the smallest percentage). Further statistical results on the first symptoms of children are given in Figure 5(b). It indicates that the percentage of children with cough as the first symptom was the highest $(92.45 \%)$, followed by the percentage of children with unsmooth breathing $(76.42 \%)$ and the percentage of children with convulsions which was the lowest (0.94\%).

3.5. Analysis of CT and Ultrasound Diagnosis Results of Children. After the children were diagnosed by the CT, it was found that there were a total of 64 children (60.38\%) with CAP, and the remaining 42 cases (39.62\%) suffered from lower respiratory infection instead of CAP. Figure 6 illustrates the further statistical analysis of the difference in the ultrasound examination results of CAP and non-CAP children. It suggests that $64.15 \%$ of children with CAP suffered from lung consolidation, while children with non-CAP did not suffer from it, and there was a very obvious difference between the two groups $(P<0.01) .59 .42 \%$ of children with CAP had abnormal pleural lines, while non-CAP children did not, and the difference was extremely obvious $(P<0.01)$. The percentage of children with pleural effusion among children with CAP and children without CAP was $6.60 \%$ and $5.66 \%$, respectively, and there was no observable difference $(P>0.05)$. The percentages of children with lung consolidation + abnormal pleural line in the two groups were extremely and dramatically different from each other $(P<0.01)$.

3.6. Comparison of the Characteristics of the Two Imaging Examinations. The ultrasound and CT examination characteristics of all children with CAP were compared and analyzed, and the results are illustrated in Figure 7. After the ultrasound and CT examinations, there was no obvious difference in lung consolidation, interstitial changes, pleural effusion, and pulmonary atelectasis $(P>0.05)$. There was observable difference in the percentage of children with abnormal pleural line, bronchial sign, bronchiectasis, lymphadenopathy, and bronchial/vascular malformation in the results of ultrasound and CT $(P<0.05)$.

3.7. Comparison of Different Imaging Characteristics in Children with CAP. The ultrasound examination result was taken as the reference to compare the imaging characteristics of the ultrasound examination. The results shown in Figure 8 


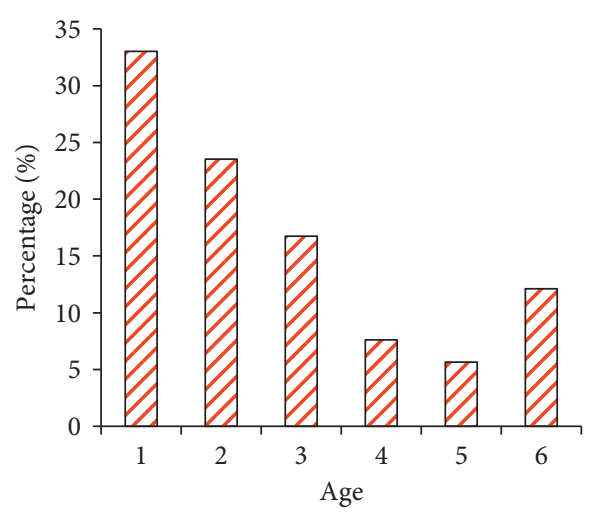

(a)

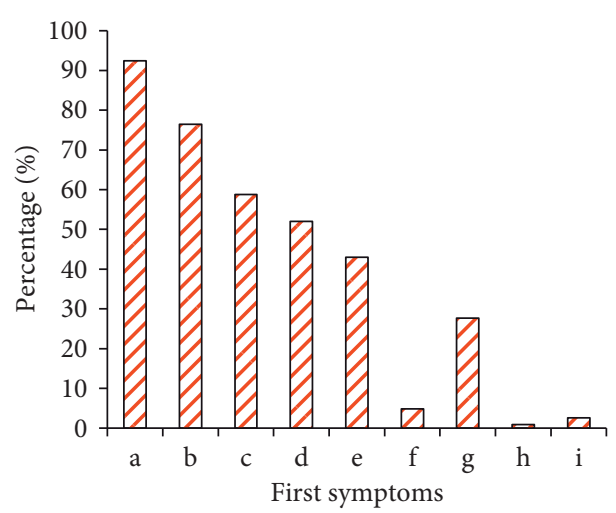

(b)

Figure 5: General information of patients. (a) The age distribution of children; 1, 2, 3, 4, 5, and 6 in the horizontal coordinate refer to 29 days -3 months, 3-6 months, 6-9 months, 9-12 months, 1-2 years, and 2-3 years old, respectively. (b) The statistical results of the first symptom of children; $\mathrm{a}, \mathrm{b}, \mathrm{c}, \mathrm{d}, \mathrm{e}, \mathrm{f}, \mathrm{g}$, h, and i in the horizontal coordinate refer to cough, unsmooth breathing, poor spirit, fever, wheezing, oliguria, poor diet, convulsions, and consciousness disorder, respectively.

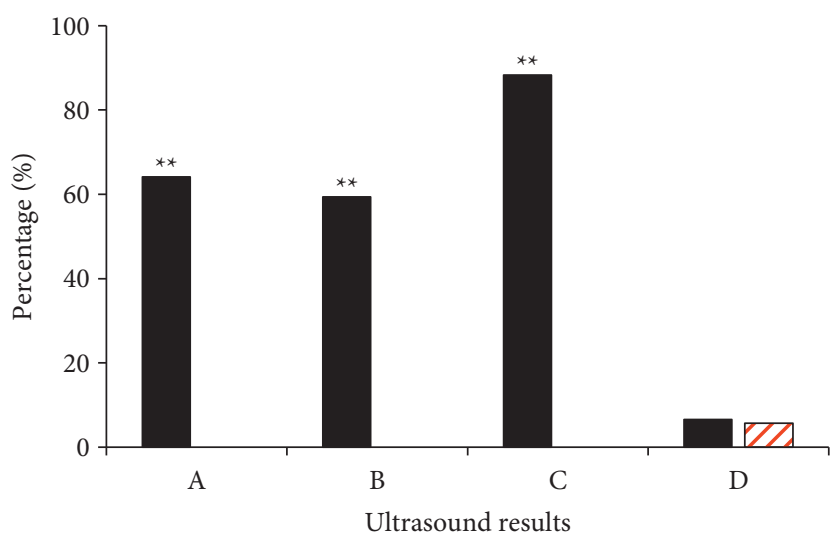

CAP group

Non-CAP group

Figure 6: Analysis of ultrasound diagnosis of the children. (a-d) The percentage of lung consolidation, abnormal pleural line, lung consolidation + abnormal pleural line, and pleural effusion, respectively. ${ }^{* *} P<0.01$ in contrast to non-CAP group.

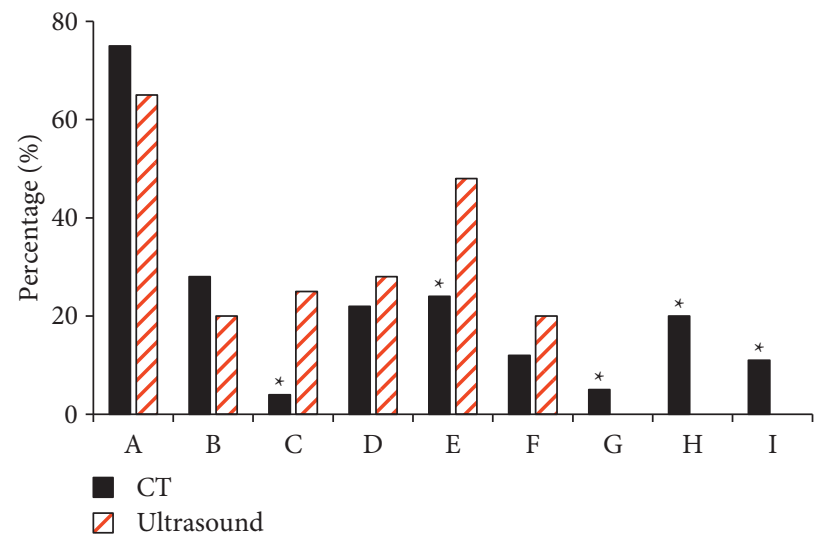

FIGURE 7: Comparison of examination characteristics of ultrasound and CT images. Note. A, B, C, D, E, F, G, H, and I in the horizontal coordinate refer to lung consolidation, interstitial changes, abnormal pleural line, pleural effusion, bronchial sign, atelectasis, bronchiectasis, lymphadenopathy, and bronchi/vascular malformations, respectively. ${ }^{*} P<0.05$ in contrast to the results of ultrasound examination. 


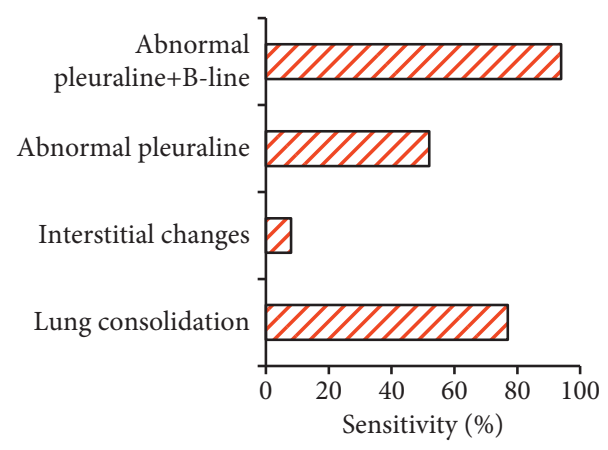

(a)

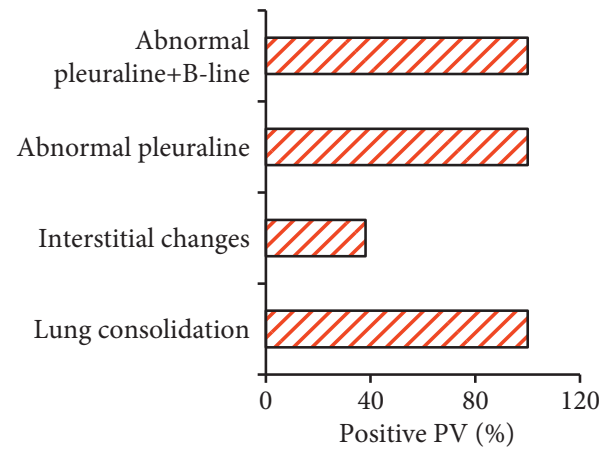

(c)

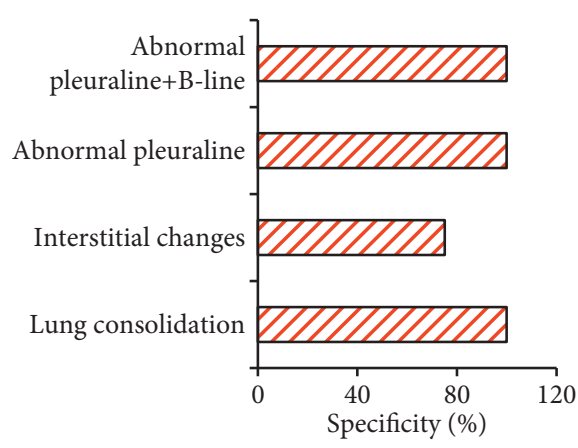

(b)

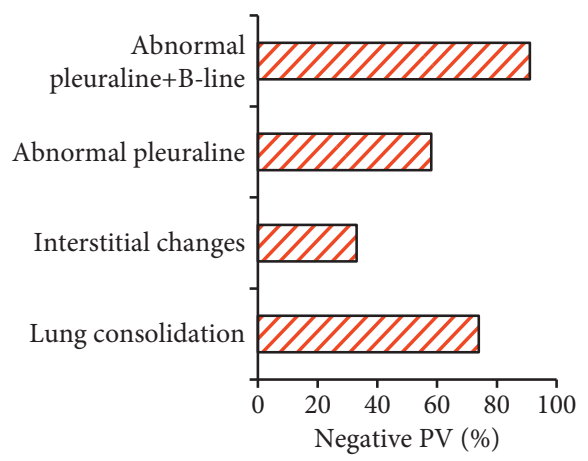

(d)

FIGURE 8: Comparison of imaging results: (a) sensitivity, (b) specificity, (c) positive PV, and (d) negative PV of the CT examination after ASiR reconstruction.

suggest that the CT detection had the highest sensitivity $(93.75 \%)$ in the pleural line abnormality + B-line/consolidation feature after ASiR reconstruction; in addition, the specificity and the positive PP were both $100 \%$ in the lung consolidation, abnormal pleural line, and abnormal pleural line $+\mathrm{B}$-line/consolidation detection. The highest negative PP was $90.63 \%$ for the abnormal pleural line + B-line/consolidation, and the lowest negative PV of interstitial changes was $34.38 \%$. Further analysis of the diagnostic results of ultrasound and CT revealed that the diagnostic results of ultrasound and CT were consistent to the greatest degree (kappa $=0.961$, and $P<0.01$ ).

\section{Discussion}

The ASiR technology is a new iterative algorithm established in the projection and image data space [12]. After iterative processing of the images obtained by the filtered back projection algorithm, the image noise is reduced, and the image resolution and image quality are improved [13]. The study of Ludwig et al. (2019) et al. [14] found that when the CT image quality remained unchanged, the amount of scanning agent used in the diagnosis of the ASiR algorithm was $46 \%$ lower than that of the FBP algorithm. The key to improving the image quality relies on the degree of noise reduction [15], which is represented by the ASiR value [16]. In this study, the ASiR technology was adopted to reconstruct and analyze the results of CT images. When the ASiR weight was $10 \%$, the noise of the image reached the maximum value $(29.87 \pm 2.93 \mathrm{HU})$; the ASiR weight had an observably negative correlation with the image noise ( $r=0.8671$, and $P<0.001)$. The maximum $C N R$ value of the image was $17.23 \pm 4.41 \mathrm{HU}$ when the ASiR weight was $100 \%$, and the CNR value had an obviously positive correlation with the ASiR weight $(r=0.917$, and $P<0.001)$. The image noise was relatively high when the ASiR weight was 40-60\%; furthermore, the lower the image noise, the higher the image quality. Thus, ASiR technology could improve the image quality by reducing noise. De Marco et al. (2018) [17] adopted the FBP algorithm and the ASiR algorithm to scan the patient's chest with $50 \%$ ASiR of conventional dose CT and found that the image noise reconstructed using conventional dose CT of $50 \%$ ASiR was similar to that of conventional dose, but the scanning dose was greatly reduced by $50 \%$, which was similar to the results of this study.

Antibiotic treatment within 4 hours after admission of CAP patients can greatly reduce the mortality rate [18]. Therefore, the use of imaging is of great significance for the early and rapid diagnosis of CAP. The imaging means for the early diagnosis of CAP mainly include X-ray, CT, and ultrasound [19]. The diagnostic accuracy of X-ray is lower than that of CT visibly, and its false positive and false negative diagnosis rates reach about $30 \%$ [20]. CT examination is the "gold standard" of CAP diagnosis. The ionizing radiation in the examination process is harmful to patients, so pregnant women and children should not be scanned with the CT examination as possible [21]. The ASiR algorithm in this study dramatically reduced the dose of CT examination. Comparing the diagnosis results after reconstruction with the results of ultrasound diagnosis, we found that the 
sensitivity of CT was $93.75 \%$ in detecting the abnormal pleural line $+\mathrm{B}$-line/consolidation feature after ASiR reconstruction. Studies have found [22] that there is a difference in the diagnostic coincidence rate of ultrasound and CT for interstitial lung disease $(P<0.05)$. Results in this study proved that there was no huge difference in the percentage of pulmonary consolidation, interstitial change, pleural effusion, and pulmonary atelectasis after ultrasound and CT examination $(P>0.05)$. The results of this study were contrary to those of Claessens et al., which may be due to the different methods used in the image reconstruction of the two. It is further shown that the diagnosis rate of lung interstitial changes based on the ASiR algorithm was dramatically improved in this study.

\section{Conclusion}

The optimal weight range of ASiR was analyzed and applied to the diagnosis of CAP in children, and the difference in the results of CAP diagnosis and ultrasound diagnosis was detected and analyzed based on ASiR in the optimal weight range. However, there are still some shortcomings in this study. It only optimized the optimal weight range of the ASiR algorithm from the objective results and failed to compare and analyze the image quality under the optimal weight range with the image quality of various algorithms such as FBP. In the future, the image quality under the optimal weight range of the ASiR algorithm will be compared with the quality of algorithms such as FBP and analyzed. In summary, the sensitivity, specificity, positive PV, and negative PV of ASiR-based chest CT in the diagnosis of CAP were close to those of ultrasound, which provided a reference basis for clinical application of ASiR algorithm to lung CT reconstruction images.

\section{Data Availability}

The data used to support the findings of this study are available from the corresponding author upon request.

\section{Conflicts of Interest}

The authors declare no conflicts of interest.

\section{References}

[1] A. K. C. Leung, A. H. C. Wong, and K. L. Hon, "Communityacquired pneumonia in children," Recent Patents on Inflammation \& Allergy Drug Discovery, vol. 12, no. 2, pp. 136-144, 2018.

[2] C. W. Lanks, A. I. Musani, and D. W. Hsia, "Communityacquired pneumonia and hospital-acquired pneumonia," Medical Clinics of North America, vol. 103, no. 3, pp. 487-501, 2019.

[3] D. M. le Roux and H. J. Zar, "Community-acquired pneumonia in children - a changing spectrum of disease," Pediatric Radiology, vol. 47, no. 11, pp. 1392-1398, 2017.

[4] A. Yazici and H. Er, "The correlation of computed tomography in the evaluation of septoplasty patients," Nigerian Journal of Clinical Practice, vol. 22, no. 9, pp. 1196-1200, 2019.
[5] T. Soma, J. Ishioka, H. Tanaka, Y. Matsuoka, K. Saito, and Y. Fujii, "Potential for computer-aided diagnosis using a convolutional neural network algorithm to diagnose fat-poor angiomyolipoma in enhanced computed tomography and T2weighted magnetic resonance imaging," International Journal of Urology, vol. 25, no. 11, pp. 978-979, 2018.

[6] A. J. Procter and J. Jacob, "Visual vs. computer-based computed tomography analysis for the identification of functional patterns in interstitial lung diseases," Current Opinion in Pulmonary Medicine, vol. 25, no. 5, pp. 426-433, 2019.

[7] P. Barca, M. Giannelli, M. E. Fantacci, and D. Caramella, "Computed tomography imaging with the adaptive statistical iterative reconstruction (ASIR) algorithm: dependence of image quality on the blending level of reconstruction," Australasian Physical \& Engineering Sciences in Medicine, vol. 41, no. 2, pp. 463-473, 2018.

[8] C. P. Upchurch, C. G. Grijalva, R. G. Wunderink et al., "Community-acquired pneumonia visualized on CT scans but not chest radiographs," Chest, vol. 153, no. 3, pp. 601-610, 2018.

[9] A. Torres, J. D. Chalmers, C. S. Dela Cruz et al., "Challenges in severe community-acquired pneumonia: a point-of-view review," Intensive Care Medicine, vol. 45, no. 2, pp. 159-171, 2019.

[10] J. A. M. Stadler, S. Andronikou, and H. J. Zar, "Lung ultrasound for the diagnosis of community-acquired pneumonia in children," Pediatric Radiology, vol. 47, no. 11, pp. 1412-1419, 2017.

[11] A. Kaysin and A. J. Viera, "Community-acquired pneumonia in adults: diagnosis and management," American Family Physician, vol. 94, no. 9, pp. 698-706, 2016.

[12] W. K. Han, J. C. Na, and S. Y. Park, "Low-dose CT angiography using ASiR-V for potential living renal donors: a prospective analysis of image quality and diagnostic accuracy," European Radiology, vol. 30, no. 2, pp. 798-805, 2020.

[13] L.-h. Chen, C. Jin, J.-y. Li et al., "Image quality comparison of two adaptive statistical iterative reconstruction (ASiR, ASiR$\mathrm{V)}$ algorithms and filtered back projection in routine liver CT," British Journal of Radiology, vol. 91, no. 1088, Article ID 20170655, 2018.

[14] M. Ludwig, E. Chipon, J. Cohen et al., "Detection of pulmonary nodules: a clinical study protocol to compare ultralow dose chest CT and standard low-dose CT using ASIR-V," BMJ Open, vol. 9, no. 8, Article ID e025661, 2019.

[15] S. Tang, X. Liu, L. He, Y. Zhou, and Z. Cheng, "Application of ASiR in combination with noise index in the chest CT examination of preschool-age children," La radiologia medica, vol. 124, no. 6, pp. 467-477, 2019.

[16] H. G. Kim, H.-J. Lee, S.-K. Lee, H. J. Kim, M.-J. Kim, and C. T. Head, "Head CT: image quality improvement with ASIR-V using a reduced radiation dose protocol for children," European Radiology, vol. 27, no. 9, pp. 3609-3617, 2017.

[17] P. De Marco and D. Origgi, "New adaptive statistical iterative reconstruction ASiR-V: assessment of noise performance in comparison to ASiR," Journal of Applied Clinical Medical Physics, vol. 19, no. 2, pp. 275-286, 2018.

[18] S. Andronikou, P. Goussard, and E. Sorantin, "Computed tomography in children with community-acquired pneumonia," Pediatric Radiology, vol. 47, no. 11, pp. 1431-1440, 2017.

[19] J. Le Bel, T. Pelaccia, P. Ray et al., "Impact of emergency physician experience on decision-making in patients with suspected community-acquired pneumonia and undergoing 
systematic thoracic CT scan," Emergency Medicine Journal, vol. 36, no. 8, pp. 485-492, 2019.

[20] Q. Zhang, Y. Ju, Y. Ma, and T. Wang, "N-acetylcysteine improves oxidative stress and inflammatory response in patients with community acquired pneumonia," Medicine, vol. 97, no. 45, Article ID e13087, 2018.

[21] T. Welte, M. Kantecki, G. G. Stone, and J. Hammond, "Ceftaroline fosamil as a potential treatment option for Staphylococcus aureus community-acquired pneumonia in adults," International Journal of Antimicrobial Agents, vol. 54, no. 4, pp. 410-422, 2019.

[22] Y.-E. Claessens, M.-P. Debray, F. Tubach et al., "Early chest computed tomography scan to assist diagnosis and guide treatment decision for suspected community-acquired pneumonia," American Journal of Respiratory and Critical Care Medicine, vol. 192, no. 8, pp. 974-982, 2015. 